\title{
Multiple Components of Delayed Potassium Current in Peptidergic Neurons of Aplysia: Modulation by an Activator of Adenylate Cyclase
}

\author{
J. A. Strong and L. K. Kaczmarek \\ Departments of Pharmacology and Physiology, Yale University School of Medicine, New Haven, Connecticut 06510
}

The neurosecretory bag cell neurons of the mollusk, Aplysia, control egg-laying behavior in the animal. In these cells, elevation of $\mathrm{cAMP}$ greatly enhances the height and width of action potentials. A similar enhancement of action potentials is seen during the bag cell afterdischarge, a $30 \mathrm{~min}$ period of repetitive activity that may be triggered by peptides from the reproductive tract or by brief extracellular stimulation. The enhancement of action potentials during an afterdischarge is well correlated with the observed elevations of cAMP. In the present study, we have examined the effects of forskolin (an activator of adenylate cyclase) and theophylline (a phosphodiesterase inhibitor) on the delayed outward currents that are likely to control repolarization of the action potential. Isolated bag cell neurons, maintained in primary culture, were studied with a whole-cell patch clamp technique. High intracellular concentrations of EGTA were used to block potassium current activated by calcium entry. Analysis of the remaining voltage-dependent delayed outward current revealed two major components, which could be separated on the basis of their different kinetic properties. Both currents $\left(I_{\mathrm{K} 1}\right.$ and $\left.I_{\mathrm{K} 2}\right)$ were carried by potassium. $I_{\mathrm{K} 1}$, which did not inactivate during $100 \mathrm{msec}$ depolarizations, was reduced in amplitude by application of forskolin and theophylline. $I_{\mathrm{K} 2}$, a current defined by its faster kinetic properties, partially inactivated during $100 \mathrm{msec}$ depolarizations. This inactivation was markedly speeded by application of forskolin and theophylline. It is suggested that such changes in outward current in response to cAMP could explain the enhancement of spike width seen during an afterdischarge in vivo.

Changes in the shape of action potentials have been observed in many types of neurons in response to synaptic or hormonal stimulation. An increase in the width or height of an action potential occurring at the nerve terminal may increase calcium influx and, hence, transmitter release. Similar changes in the soma action potential may influence metabolic functions, such as protein synthesis. One important determinant of action potential width is the delayed outward current, which is responsible for the repolarization of the action potential in most excitable tissues. We have studied the regulation of delayed outward current by cAMP-dependent mechanisms in the bag cell neurons of Aplysia.

The peptidergic bag cell neurons provide an interesting system in which to study the biochemical modulation of a neuron's electrical properties. These neurosecretory cells, which control

\footnotetext{
Received Apr. 24, 1985; accepted July 18, 1985.

This work was supported by Grant NS-18492 and a Klingenstein Fellowship to L.K.K. and PHS Research Fellowship NS-07125 to J.A.S.

Correspondence should be addressed to J. A. Strong, Department of Pharmacology, Yale University School of Medicine, 333 Cedar Street, New Haven, CT 06510.

Copyright $@ 1986$ Society for Neuroscience $0270-6474 / 86 / 030814-09 \$ 02.00 / 0$
}

the animal's egg-laying behavior, can undergo several profound and long-lasting modifications of their electrical properties. Normally, the cells have high resting potentials and show no spontaneous activity. However, stimulation of the adjacent connective nerves, or application of neuroactive peptides from the reproductive tract, causes the cells to depolarize and fire repetitively for $30 \mathrm{~min}$. During this afterdischarge, there are multiple changes in the cells' electrical properties, including a marked enhancement of the height and width of action potentials. These changes in the action potential are likely to increase calcium influx and hence the release of bag cell peptides during the afterdischarge. In turn, these peptides act on other neurons and on the reproductive tract to orchestrate a complex pattern of behavior, culminating in egg-laying (Heller et al., 1980; Kupferman and Kandel, 1970; Strumwasser et al., 1980).

The bag cell neurons form two clusters of 200-400 homogeneous cells, and can thus be studied (in isolation from all other neurons) with conventional biochemical techniques. In addition, the cells are large enough $(35-150 \mu \mathrm{m})$ to permit electrophysiological recordings. Both techniques have been brought to bear in attempting to understand the mechanisms of long-lasting changes in excitability, such as the afterdischarge and refractoriness. To date, most studies have focused on the cAMP- and calcium-dependent protein kinases.

Stimulation of the bag cell clusters increases their cAMP content, and elevations of cAMP have been shown to play an important role in the initiation and maintenance of the natural afterdischarge. In addition, afterdischarges may be induced with drugs that elevate cAMP (Kaczmarek et al., 1978; Kauer and Kaczmarek, 1985). We have therefore been interested in studying the effects of cAMP elevations on the cells' electrical properties. In a two-microelectrode voltage clamp study of bag cell neurons maintained in primary culture, Kaczmarek and Strumwasser (1984) found that external application of cAMP analogs had several effects, including reduction of net outward current during depolarizing voltage clamp steps, the appearance of a region of negative slope resistance at potentials near the resting potential, and the reduction of the transient outward current (the A-current). Strong (1984), using a whole-cell patch clamp technique, found that applications of the adenylate cy. clase activator, forskolin, reduced the A-current by speeding up its inactivation kinetics.

In order to better characterize the reduction of net outward current observed by Kaczmarek and Strumwasser (1984), we have used the whole-cell patch clamp technique (Hamill et al., 1981). This technique allows experimental control over the composition of smaller ions in the cytoplasm and, hence, is useful for separating some of the multiple currents present in these cells. In the present study, we have used high internal EGTA concentrations to block calcium-activated potassium currents, and have studied the effects of forskolin on the re- 
maining delayed outward current. We report that the delayed outward current in these cells is comprised of two kinetically distinct components, which we have termed $I_{\mathrm{K} 1}$ and $I_{\mathrm{K} 2}$. Forskolin application reduces the amplitude of one of these currents $\left(I_{\mathrm{K} 1}\right)$ and causes the second current $\left(I_{\mathrm{K} 2}\right)$ to inactivate more rapidly. We conclude that all threc of the major voltage-dependent potassium currents in these cells $\left(I_{\mathrm{K} 1}, I_{\mathrm{K} 2}\right.$, and the A-current) may be regulated by cAMP-dependent mechanisms.

\section{Materials and Methods}

\section{Animals and cell culture}

Aplysia californica were obtained from Alacrity Marine Services (Redondo Beach, CA) and kept at $14^{\circ} \mathrm{C}$. The methods used for primary culture of the bag cell neurons were similar to those described by Kaczmarek and Strumwasser (1981). To summarize: The abdominal ganglion was removed from the animal and incubated at room temperature in a solution of neutral protease $(1.25 \%$ for $18 \mathrm{hr}$ or $2.5 \%$ for $6 \mathrm{hr})$; the bag cell cluster was isolated and desheathed; and the cells were triturated and seeded into plastic petri dishes (Falcon) containing modified Eagle's medium (MEM; see below). The petri dishes were pretreated with the following solutions: $0.1 \mathrm{~N} \mathrm{HCl}(30 \mathrm{~min})$, followed by $0.1 \mathrm{~N} \mathrm{NaOH}(30$ min), followed by MEM plus $1 \mathrm{mg} / \mathrm{ml}$ BSA $(12 \mathrm{hr})$. This treatment prevented the cells from adhering to the dishes and growing processes. These "floating" cells had ionic currents similar to those of normally cultured cells, but could be internally dialyzed much more rapidly. The cells were kept at $14^{\circ} \mathrm{C}$. Experiments were performed at room temperature $\left(21-25^{\circ} \mathrm{C}\right)$ on cells that had been in primary culture for 1 or $2 \mathrm{~d}$.

\section{Voltage clamp and intracellular dialysis}

A whole-cell patch clamp technique (Hamill et al., 1981), modified for use in larger cells as described in Strong (1984), was used. The virtualground type voltage clamp had a $100 \mathrm{M} \Omega$ feedback resistor. Two modifications were made in order to achieve faster internal dialysis than was seen by Strong (1984). First, "floating" cells, as described above, were used. Second, smaller bag cell neurons were used. This was achieved by using smaller ( $150 \mathrm{gm}$ ) Aplysia. Cell diameters were 30-60 $\mu \mathrm{m}$, and dialysis pipettes had inner diameters of $5-10 \mu \mathrm{m}$. With these modifications, exchange of small ions (e.g., potassium channel blockers) between the pipette and the cytoplasm occurred on a time scale of 1-4 $\min$.

\section{Data acquisition and analysis}

Currents were amplified and filtered at $4000 \mathrm{~Hz}$. A PDP 11/23 laboratory computer was used to record and store the data, to control the voltage stimuli, and to analyze the data. In each record, the current required to clamp the cell to the holding potential was subtracted. In some records, linear leak subtraction was used; this is noted in the figure legends. A holding potential of $-60 \mathrm{mV}$ was used unless otherwise noted. Some current records werc fitted with the sum of two exponentials; this was done by eye, as described by Strong (1984).

\section{Solutions}

The artificial seawater (ASW) had the following composition: $\mathrm{NaCl}, 460$ $\mathrm{mm}$; KCl, $10.4 \mathrm{~mm}$; HEPES, $15 \mathrm{~mm}$; $\mathrm{CaCl}_{2}, 11 \mathrm{~mm}$; $\mathrm{MgCl}_{2}, 27 \mathrm{~mm}$; $\mathrm{MgSO}_{4}, 28 \mathrm{~mm}$; glucose, $1 \mathrm{mg} / \mathrm{ml}$ (pH adjusted to 7.8 ). The culture medium (MEM) was made by adding essential and nonessential amino acids, vitamins, glutamine, penicillin, and streptomyocin (Gibco) to the ASW (Kaczmarek and Strumwasser, 1981). Recordings were made in MEM unless otherwise noted. The internal solution used in the dialysis pipette contained $570 \mathrm{~mm} \mathrm{~K}$ aspartate, $10 \mathrm{~mm}$ HEPES, $10 \mathrm{~mm}$ reduced glutathione, $5 \mathrm{~mm} \mathrm{MgCl}, 1 \mathrm{mg} / \mathrm{ml}$ glucose, $5 \mathrm{~mm}$ ATP (Sigma, grade II disodium salt), $0.1 \mathrm{~mm}$ GTP (Sigma, type 3 disodium salt), $20 \mathrm{~mm}$ EGTA, $4.14 \mathrm{CaCl}_{2}$ (pH adjusted to 7.3). MEM and the internal solution were kept frozen until use. All solutions were filtered $(0.2 \mu \mathrm{m})$ immediately before use. Forskolin was obtained from Calbiochem. Stock solutions of 10 or $20 \mathrm{~mm}$ in ethanol were made. Stock solutions of theophylline were $10 \mathrm{~mm}$ in ASW. Aliquots of the stock solutions of the drugs were added to the bath to give the final concentrations listed in the text. Control applications of ethanol had no effect on the cells.
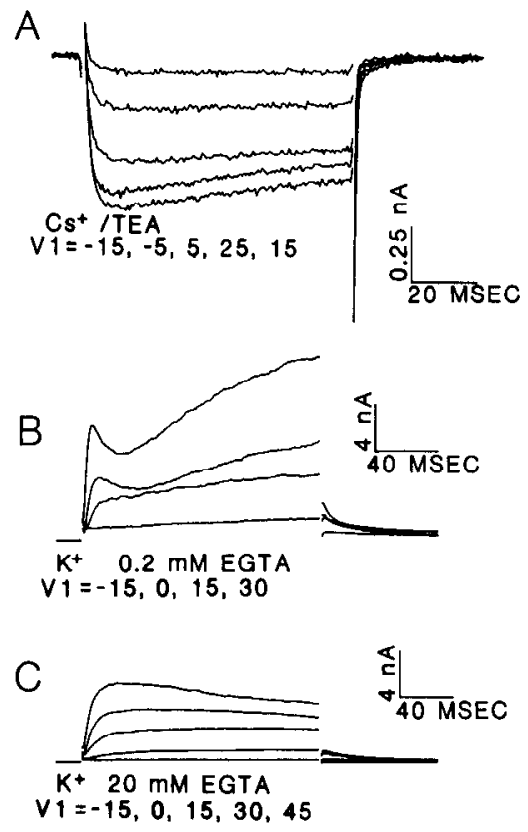

Figure 1. Ionic currents in dialyzed bag cell neurons. A, Inward currents seen in a cell dialyzed with internal solution (see Materials and Methods) in which potassium was replaced with cesium (470 mM) and TEA $(100 \mathrm{~mm})$. Currents shown were evoked by depolarizations to -15 (smallest current), $-5,5,25$, and 15 (largest current) $\mathrm{mV}$, followed by repolarization to $-40 \mathrm{mV}$. The ohmic portion of the leakage conductance, as measured during small hyperpolarizations, has been subtracted. $B$, Outward currents seen in another cell, dialyzed with internal solution in which the usual $20 \mathrm{~mm}$ EGTA, $4.14 \mathrm{~mm} \mathrm{CaCl}_{2}$ had been replaced with $0.2 \mathrm{~mm}$ EGTA, with no added calcium. As in $A$, currents were evoked by depolarization to the indicated potentials, followed by repolarization to $-40 \mathrm{mV}$. $C$, Outward currents seen in another cell dialyzed with the internal solution described in Materials and Methods, using a vollage protocol as in $B$.

\section{Results}

The dialyzed, cultured bag cell neurons contain several distinct ionic currents, most of which are similar to those that have been described previously in other molluscan neurons (Adams et al., 1980). Some of these currents may be isolated or eliminated, depending on the composition of the internal solution.

Inward currents are revealed when $\mathrm{Cs}^{+}$and $\mathrm{TEA}^{+}$ions are substituted for $\mathrm{K}^{+}$in the internal solution (Fig. 1A). These currents are carried predominantly by calcium: They persist when external sodium is replaced by $N$-methylglucamine, may be carried by barium, and are blocked by $\mathrm{La}^{3+}, \mathrm{Co}^{2+}$, and removal of extracellular calcium. As has been reported by others (e.g., see Kostyuk, 1984), the calcium current is sensitive to internal dialysis and could rarely be recorded for more than 30-40 min. A minority of cells also contains a small, rapidly inactivating sodium current (Kaczmarek and Strumwasser, 1984; examples not shown).

Figure $1 B$ shows the pattern of predominantly outward currents seen when the internal solution contains potassium aspartate, and in which the concentration of the calcium buffer, EGTA, is reduced from 20 to $0.2 \mathrm{~mm}$. The outward current has a rapidly activating component, which is followed by a second, more slowly developing, component. This slower component resembles the calcium-activated potassium current that has been observed in other molluscan neurons (Adams et al., 1980). Several pieces of evidence suggest that the slowly developing outward current in the bag cell neurons is also calcium-activated. This component disappears when the internal solution contains higher concentrations of EGTA (Fig. 1C), is blocked by $\mathrm{La}^{3+}$, $\mathrm{Co}^{2+}$, and removal of extracellular calcium; like the calcium 
A
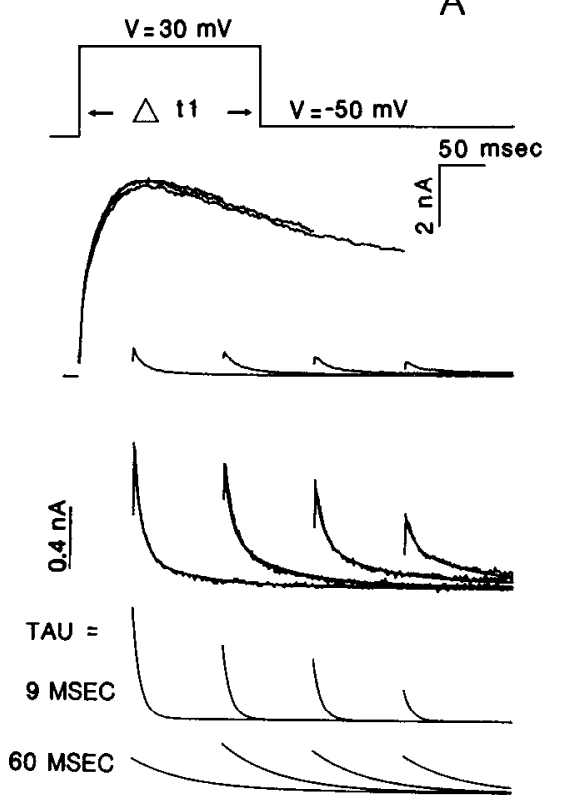

B

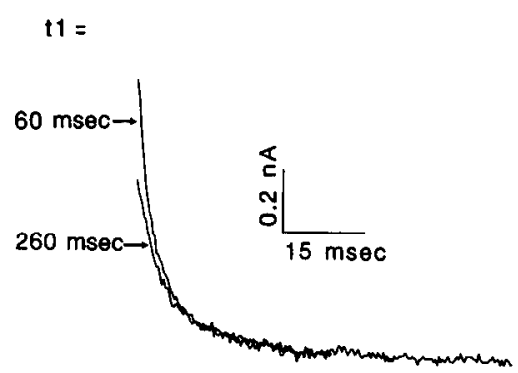

Figure 2, Outward current has two kinetically distinct components. $A$, Currents during depolarization to $30 \mathrm{mV}$ lasting $60,160,260$, and $360 \mathrm{msec}$ (followed by repolarization to $-50 \mathrm{mV}$ ) are shown superimposed. The tail currents seen during the repolarizations are replotted below at higher gain. Tail currents have been fitted with the sum of two exponentials; the fit is superimposed on the data. The individual components of the fitted function are displayed separately beneath. The first two data points of the tail currents $(2 \mathrm{msec})$ containing the capacitative transient have been omitted. The two time constants were constrained to have the values fitted to the first tail current record. $B$, The tail currents following the first $(60 \mathrm{msec})$ and third $(260 \mathrm{msec})$ depolarizations in $A$ are also shown superimposed. current, it is also removed simply by prolonged $(30-40 \mathrm{~min})$ intracellular dialysis.

Figure $1 C$ shows the pattern of currents in cells dialyzed with an internal solution containing potassium aspartate and $20 \mathrm{~mm}$ EGTA (as described in Materials and Methods). The currents are predominantly outward (though a small, early, net inward current can be detected in some cells), are rapidly activated at potentials above $0 \mathrm{mV}$, and contain an inactivating component whose rate of inactivation increases sharply with increasing potential. The currents appear to be independent of voltage-activated calcium entry; a similar pattern of outward current is seen after addition of $100 \mu \mathrm{M} \mathrm{La}{ }^{3+}$ (though this ion causes shifts in the voltage dependence of some of the outward currents). In contrast to the calcium and calcium-activated currents, stable outward currents such as those in Figure $1 C$ could sometimes be recorded for up to $90 \mathrm{~min}$.

This internal solution, containing potassium aspartate and 20 mM EGTA (Fig. 1C), was used to investigate the voltage-dependent outward currents in the bag cell neurons. As described above, this solution eliminates the slowly developing outward current activated by calcium entry. The A-current (transient outward current) is eliminated by using holding potentials of -60 to $-50 \mathrm{mV}$. At a holding potential of $-60 \mathrm{mV}$, over $99 \%$ of the A-current is inactivated in these cells (Strong, 1984).

\section{Voltage-dependent outward current in bag cell neurons has two components}

Figure 2 shows that the outward current present in the cells dialyzed with high concentrations of EGTA contains two kinetically distinct components. The figure shows the tail currents that appear when the membrane is repolarized to $-50 \mathrm{mV}$ following steps to $+30 \mathrm{mV}$ of varying durations. If this experiment were done in a neuron or axon that contained only a delayed rectifier current, such as that described by Hodgkin and Huxley (1952), one would expect the tail currents to be simple exponentials, representing the decay of the potassium current activated during the preceding depolarization. This is not the case in the bag cell neurons. The outward tail currents shown in Figure 2 cannot be described by a single exponential, but have both fast and slow components. (One might also expect to see an inward tail current, which would reflect the closing of calcium channels; however, these tail currents are much smaller than the outward tail currents and are too fast to be resolved clearly with the voltage clamp method used; see Fig. $1 A$.)

The outward tail currents in Figure $2 A$, shown on an expanded current scale, have been fitted with the sum of two exponentials, with time constants of 9 and $60 \mathrm{msec}$. The fitted function is superimposed on the data, and the two individual components of the fitted function are displayed separately beneath it. The data show that the fast component of the current is responsible for the inactivation of outward current during depolarizing pulses: Increasing the duration of the depolarizing pulse results in a reduction of the fast component of the tail current observed on repolarization, and this reduction parallels the decline in outward current during the depolarization. This result can also be seen without using the curve-fitting procedure. In Figure $2 B$, the tail currents from a short and a longer depolarization in Figure $2 A$ are superimposed. It is evident that the two records differ only during the first few milliseconds and that the late, slow components are superimposed.

The most parsimonious explanation of the results shown in Figure 2 is that the bag cell neurons contain two distinct voltagedependent outward currents (rather than a single current with very complex kinetics). This explanation is supported by the observation that the two components were differently affected by forskolin (see below), and by the finding that the ratio of the two components varicd considerably from cell to cell. The ratio of the slow to the fast component of the tail current at $-40 \mathrm{mV}$ (following a fixed depolarization to $30 \mathrm{mV}$ ) varied 6-fold between different cells, ranging from 0.33 to 2.0 . However, we were not able to effect a further separation of these two currents. It was not possible to completely isolate one of the currents by changing the holding potential or by restricting the range of the depolarizing pulses, because the two currents showed considerable overlap in the voltage dependence of activation and resting inactivation. Both currents were blocked by extracellular TEA (40 mM), but this agent did not prove useful for differentiating the two.

In different cells, the time constants for the tail currents at $-40 \mathrm{mV}$ ranged from 4 to $10 \mathrm{msec}$ for the fast current and from 15 to $60 \mathrm{msec}$ for the slow current. In individual cells, the two time constants had a fixed ratio; observed values of this ratio ranged from 3.5 to 7.0. The time course of activation of the two currents could not be resolved accurately through kinetic analysis of the tail currents.

Although the slow current showed little or no inactivation 


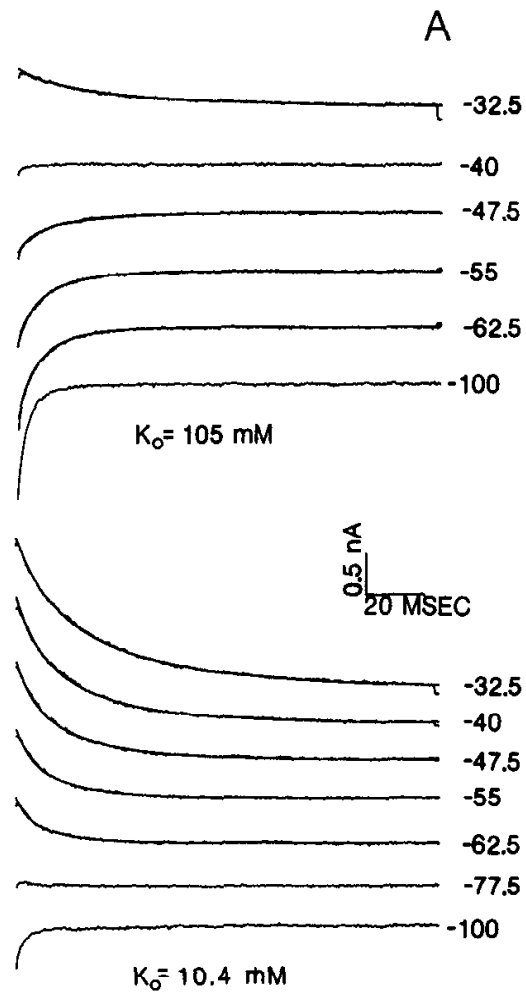

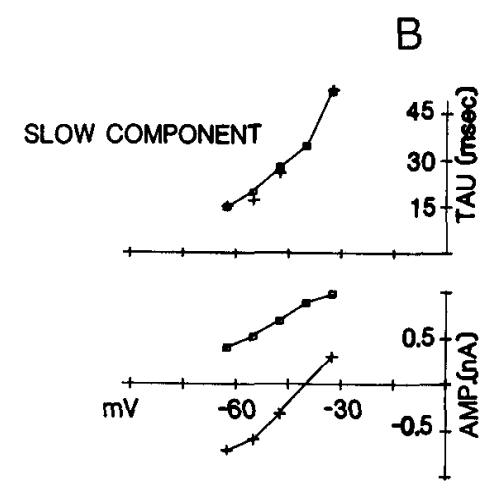

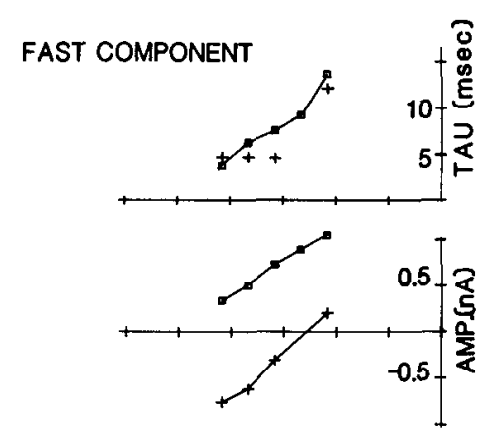

Figure 3. Tail currents in normal and elevated external potassium. $A$, Tail currents elicited by a $55 \mathrm{msec}$ depolarization to $+30 \mathrm{mV}$ (not shown), followed by repolarization to the indicated potential. Currents are displaced from one another for clarity. $\mathrm{K}_{\mathrm{o}}{ }^{+}$was $105 \mathrm{~mm}$ (top) or the normal value of $10.4 \mathrm{~mm}$ (bottom). In both sets of records, the extracellular solution also contained 5 $\mathrm{mM} \mathrm{CsCl}$. This was found to have no effect on the outward currents or tail currents, but prevented the large increase in leakage conductance otherwise seen in elevated $\mathrm{K}_{\mathrm{o}}+$. Tail currents at voltages more positive than $-65 \mathrm{mV}$ have been fitted with the sum of two exponentials; the small current seen at $-40 \mathrm{mV}$ in $105 \mathrm{~mm} \mathrm{~K}^{+}$was not fitted. The fitted functions are superimposed on the data. The first $2 \mathrm{msec}$ of each trace, containing the capacitative transient, are not shown. $B$, Amplitudes and time constants of the exponential fits shown in $A$. The amplitude and time constant of the slower of the two exponential components (at any given potential) are shown in the upper pair of graphs; parameters of the faster component are shown in the lower pair of graphs.,$+ \mathbf{K}_{\mathrm{o}}^{+}=105 \mathrm{mM} ; \square, \mathrm{K}_{\mathrm{o}}^{+}=$ $10.4 \mathrm{~mm}$.

during depolarizations such as those in Figures 1 and 2, it did partially inactivate during much longer depolarizations, i.e., depolarizations lasting 500 to $1000 \mathrm{msec}$. Because such long pulses were not used in the experiments presented here, we refer to the slow current as a noninactivating current.

\section{Both currents are carried by potassium}

Given the composition of the internal and external solutions used in these experiments, the only ions that could be carrying the outward currents are $\mathrm{Cl}^{-}, \mathrm{H}^{+}$, and $\mathrm{K}^{+} . \mathrm{Cl}^{-}$is unlikely to be a significant charge carrier, because the outward currents are unaltered when external chloride is replaced by aspartate (data not shown). Figure 3 shows that both the fast and slow currents are carried predominantly by potassium. Outward currents were activated by a brief depolarization to $+30 \mathrm{mV}$, and tail currents were elicited by repolarization to the indicated potentials. In the normal external and internal solutions $\left(\mathrm{K}_{\mathrm{o}}{ }^{\prime}=10.4 \mathrm{mM}\right.$, $\mathrm{K}_{i}^{+}=570 \mathrm{~mm}$ ), the calculated Nernst potential for potassium is $-100 \mathrm{mV}$. The tail currents did have a reversal potential below $-80 \mathrm{mV}$ in these solutions. This is much more negative than the Nernst potential for $\mathrm{H}^{+},-29 \mathrm{mV}$. However, it was difficult to measure a clear reversal potential for both the fast and slow currents for the following reasons: (1) The tail currents, especially of the fast current, became quite fast at more negative potentials; (2) inward currents in $10 \mathrm{mM} \mathrm{K}_{0}^{+}$were small; and (3) at more negative potentials, the current in these cells showed a slow, relatively voltage-insensitive relaxation, which tended to obscure the tail currents activated by depolarization (see Fig. $3 A$ ). However, as is shown in Figure $3 A$, when external potassium was elevated to $105 \mathrm{~mm}$, both the slow and the fast components of the tail currents reversed near the calculated $E_{\mathrm{K}}$ of $-42 \mathrm{mV}$. This indicates that both currents are carried predominantly by potassium. Elevating external potassium had little effect on the value of the time constants (Fig. $3 B$ ). Further characterization of the selectivities of the currents was not attempted.

\section{Both outward currents are affected by forskolin}

In order to study the effects of elevations of intracellular cAMP on the voltage-dependent currents, we used bath application of the adenylate cyclase activator, forskolin (Seamon et al., 1981), along with the phosphodiesterase inhibitor, theophylline. Figure 4 shows the pattern of outward currents seen during a series of depolarizing steps, before and $15 \mathrm{~min}$ after the addition of 50 $\mu \mathrm{M}$ forskolin and $1 \mathrm{mM}$ theophylline to the bath. The drugs caused a marked reduction in the net outward current, a speeding up of the inactivation, and a suppression of the tail currents observed during repolarization. These effects were seen in all cells studied. The same result was obtained when RO20-1724, a non-methylxanthine inhibitor of cAMP phosphodiesterase in Aplysia (Levitan and Norman, 1980), was used in place of theophylline. No effects on the outward currents could be observed

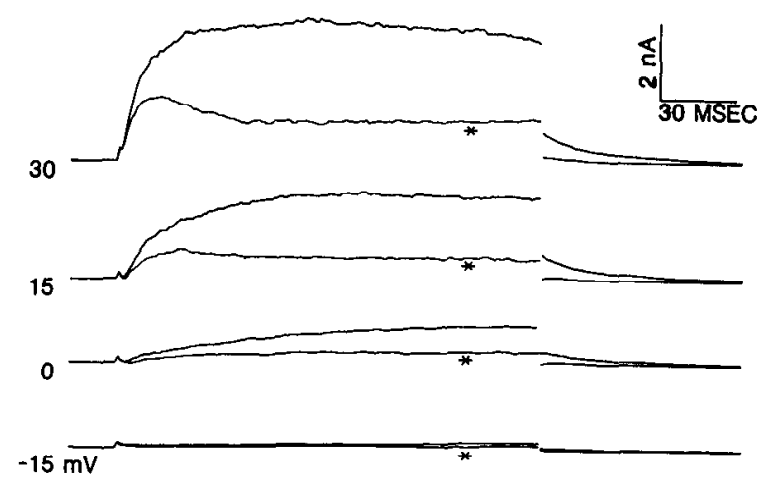

Figure 4. Effect of forskolin and theophylline on outward currents. Currents during depolarization to the indicated potentials, followed by repolarization to $-40 \mathrm{mV}$. The smaller current in each pair of traces $\left({ }^{*}\right)$ is that seen $15 \mathrm{~min}$ after addition of forskolin $(50 \mu \mathrm{M})$ and theophylline ( $1 \mathrm{~mm})$ to the bath. The larger currents at each potential were taken immediately before addition of the drugs. 
Figure 5. Time course of the forskolin effect. $A$, Currents evoked by $75 \mathrm{msec}$ steps to $+30 \mathrm{mV}$, followed by repolarization to $-40 \mathrm{mV}$. Bottom, The tail currents seen during the repolarization are expanded and displaced from each other. The time (min) at which each record was taken is indicated to the left; at "time $0, " 15 \mu \mathrm{M}$ forskolin and 1.0 mM theophylline were added to the bath. (In the upper traces, earlier times correspond to larger current traces.) The tail currents have been fitted with the sum of two exponentials. In each case the two time constants were constrained to have the same values ( 7 and $27 \mathrm{msec}$ ) that fit the earliest tail current. The fitted functions are shown superimposed on the tail currents below. $B$, Amplitudes of the slow $(+)$ and fast $(\square)$ components of the functions fitted to the tail currents in $A$, plotted as a function of time of exposure to forskolin and theophylline.

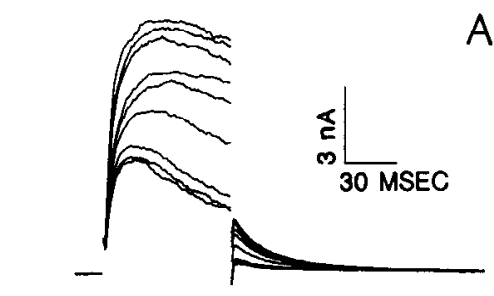

A

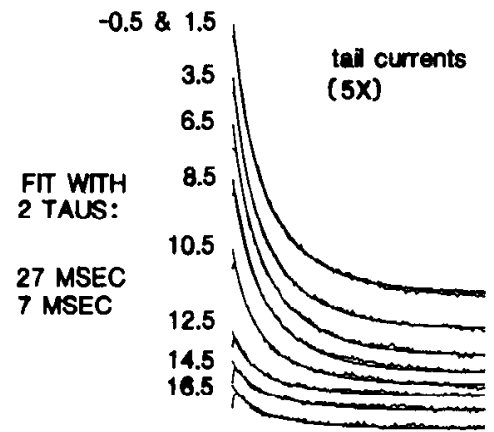

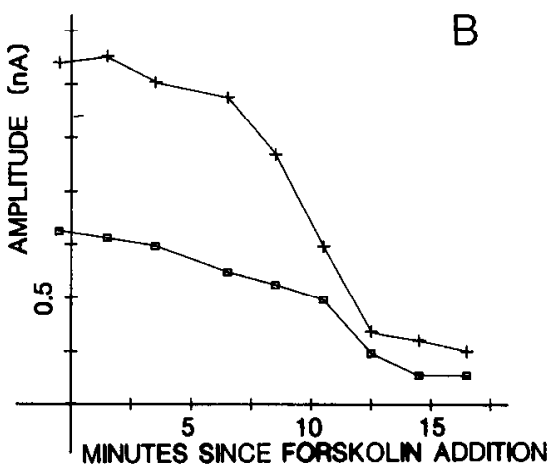

when theophylline was applied alone. Forskolin applied without a phosphodiesterase inhibitor had much smaller effects, or, in some cases, only transient effects.

The time course of the forskolin effect is shown in Figure 5, which shows the currents evoked by steps to $+30 \mathrm{mV}$ at various times after the addition to the bath of $15 \mu \mathrm{M}$ forskolin and 1 $\mathrm{mm}$ theophylline. As in Figure 2, the tail currents seen during repolarization to $-40 \mathrm{mV}$ can be fitted with the sum of two exponentials (Fig. 5A). For the first 4-6 min after addition of drugs to the bath, there was little effect on the currents. Then, during the next 5-10 min, there were sharp progressive reductions in the net outward current (Fig. 5A) and in the amplitudes of both the fast and slow components of the tail currents (Fig. $5 B)$. This indicates that both the fast and slow currents are reduced by forskolin, and that the reduction occurs without any marked change in the time constants of the tail currents.

During stimulation of an afterdischarge in intact bag cell clusters, elevation of cAMP, onset of spontaneous activity, and enhancement of action potential height and width all occur within 2-3 min following the stimulation. This suggests that elevations of cAMP can rapidly alter electrical characteristics in these cells. Since in other systems (Seamon et al., 1981), forskolin causes a rapid elevation of cAMP, the 5-15 min delay between the addition of forskolin and the reduction of outward

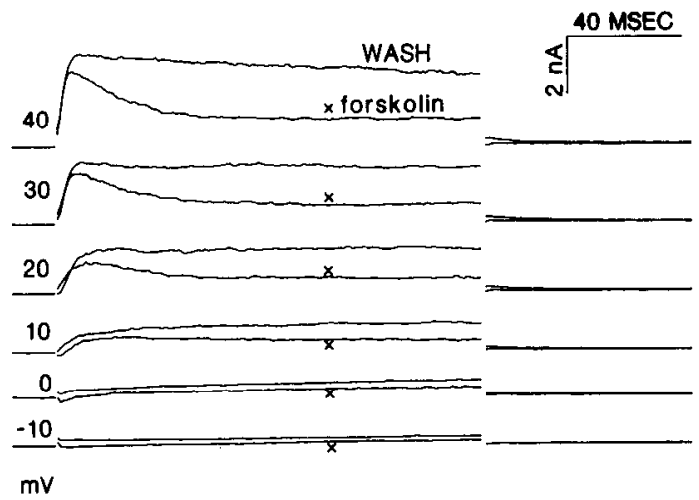

Figure 6. Reversal of the forskolin effect. Outward currents in a cell that had been pretreated with forskolin $(10 \mu \mathrm{M})$ and theophylline $(1.0$ $\mathrm{mM}$ ) for $45 \mathrm{~min}$ before beginning intracellular dialysis $(x$, smaller current in each pair of traces) and after removal of drugs from the bath (larger current in each pair). The $150 \mathrm{msec}$ depolarizations to the indicated potential were followed by repolarization to $-50 \mathrm{mV}$. current found in these experiments is somewhat puzzling. However, such a delay is characteristic of all the cAMP effects seen in cultured bag cell neurons, regardless of the method (forskolin, cAMP analogs) used to elevate cAMP. The delay is seen in cells impaled with microelectrodes as well as in dialyzed cells (Kaczmarek and Strumwasser, 1984). In the present experiments, the forskolin effects on all three outward currents - the two effects seen in Figure 5, and the effect on the A-current kinetics (Strong, 1984)-had very similar delays in any given cell. The delay could not be removed by preincubating with the phosphodiesterase inhibitor before adding the forskolin. In a minority of cells (approximately 10\%), however, a time course different from that shown in Figure 5 was seen. In these cells, a full forskolin effect was observed transiently 30-60 sec after the drugs were added to the bath. The effect disappeared within 1-3 min, then reappeared with the typical time course shown in Figure 5. This suggests that forskolin can act rapidly in the bag cell neurons, as it does in other systems. Perhaps stimulation of an afterdischarge in situ activates additional biochemical mechanisms that allow more rapid activation of the adenylate cyclase and/or cAMP-protein kinase enzyme systems.

\section{The forskolin effect is reversible}

In other systems, activation of adenylate cyclase by forskolin is a rapidly reversible process (Seamon et al., 1981). We found it difficult to record from a single dialyzed cell long enough to examine the currents before, during, and after exposure to forskolin. Instead, the procedure shown in Figure 6 was used to demonstrate that the forskolin effects were at least qualitatively reversible. The cell was pretreated with $10 \mu \mathrm{M}$ forskolin and 1 $\mathrm{mm}$ theophylline for $45 \mathrm{~min}$ before the beginning of intracellular dialysis. The outward currents recorded after such pretreatment were small and inactivated rapidly; the effects of forskolin seemed, at least qualitatively, to be the same whether the drug was added to the bath before or after the beginning of intracellular dialysis. As expected, if the effects of forskolin are reversible, the outward currents are enhanced and inactivated more slowly following removal of the drugs from the bath (Fig. 6).

\section{Forskolin causes the fast current to inactivate more rapidly}

Application of $50 \mu \mathrm{M}$ forskolin caused such a large reduction in the outward currents that it was no longer possible to resolve the tail currents into fast and slow exponential components. In order to further investigate the effects of forskolin on the two 
A

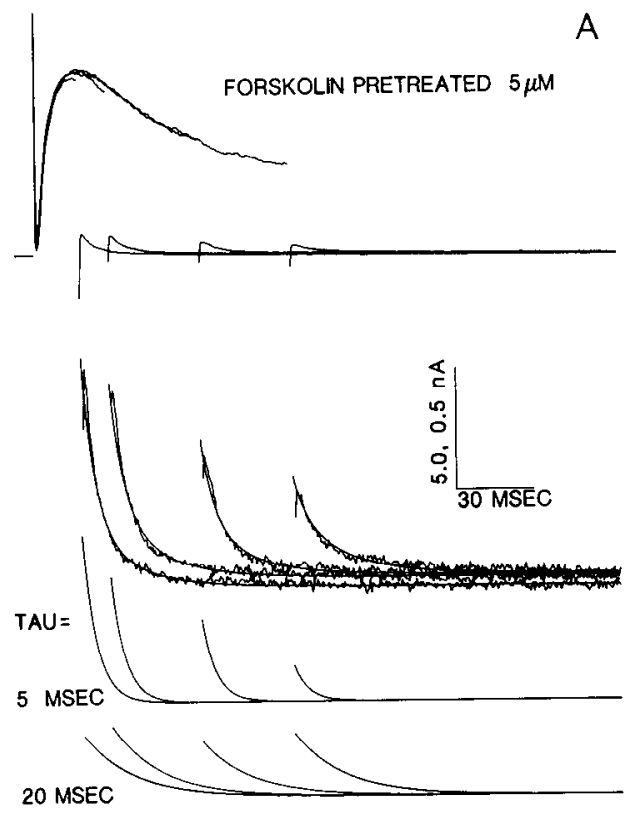

B

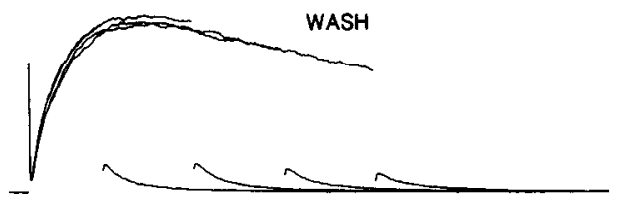

C

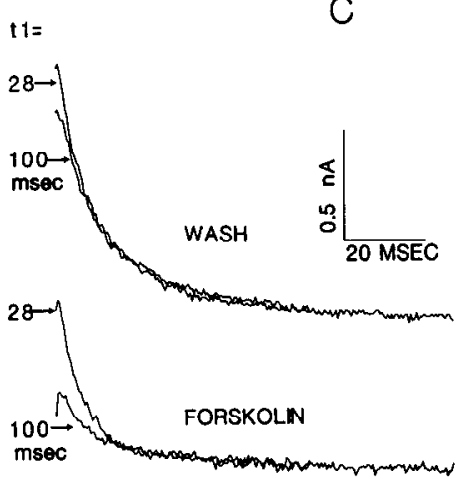

Figure 7. Inactivation of the fast component of outward current in a forskolin-pretreated cell. $A$, Currents evoked by depolarizations to $30 \mathrm{mV}$ lasting $17,28,65$, and $100 \mathrm{msec}$, followed by repolarization to $-40 \mathrm{mV}$, are shown superimposed. The tail currents at $-40 \mathrm{mV}$ are replotted below, expanded by a factor of 10 and with the first data point $(0.5 \mathrm{msec})$ omitted. Each record has been fitted with the sum of two exponentials, with time constants of 5 and $20 \mathrm{msec}$, which were determined by the fit to the first (largest) record. The fitted function is superimposed on the data; the two individual components are redrawn separately beneath. The protocol is exactly like that of Figure 2, but in this case the cell had been pretreated with $5 \mu \mathrm{M}$ forskolin and $1 \mathrm{~mm}$ theophylline for $40 \mathrm{~min}$ before beginning intracellular dialysis. $B$, Currents evoked by a voltage protocol identical to that used in $A$, showing that inactivation during the pulse to $30 \mathrm{mV}$ was slower after the forskolin and theophylline were removed from the bath. Current and time scales are the same as in $A$, top. Tail currents could still be well fitted by the sum of two exponentials with time constants of 5 and $20 \mathrm{mscc}$ (not shown). The lack of a dramatic recovery of the amplitude of the current following removal of the drugs (compare with Figure 6) may be due to a slow, constant loss of outward current that was sometimes seen in the dialyzed cells; the records in $A$ and $B$ were taken 25 min apart. $C$, Tail currents seen after 28 and $100 \mathrm{msec}$ depolarizations are superimposed. The upper pair of currents is redrawn from $B$ $(W A S H)$, the lower pair from $A(F O R S K O L I N)$. The first two data points $(1.0 \mathrm{msec})$ in each trace have been omitted.

outward currents, we studied the effects of submaximal doses of forskolin. Figure 7 shows the results of such an experiment. The protocol is exactly like that in Figure 2; tail currents seen after depolarizations to $+30 \mathrm{mV}$ of varying duration have been fitted with the sum of two exponentials. However, in this case, the cell had been pretreated with $5 \mu \mathrm{M}$ forskolin and $1 \mathrm{~mm}$ theophylline for $40 \mathrm{~min}$ before intracellular dialysis was begun. As in other forskolin-treated cells, the outward current inactivated rapidly. (When the forskolin and theophylline were subsequently removed from the bath, the outward current inactivated more slowly; see Fig. 7B.) The data in Figure 7 reveal that it is always the fast current that inactivates during a depolarization, regardless of whether this inactivation is rapid (as seen in forskolin-treated cells) or slower (as in untreated cells). These results suggest that forskolin and theophylline have two distinct effects. The fast current inactivates much more rapidly following application of the drugs, while the slow current is simply reduced in amplitude. These two effects are likely to account for the marked effects of forskolin and theophylline on the net outward current shown in Figure 4. However, the data do not rule out additional effects of forskolin, such as a reduction in amplitude of the fast current or modification of activation kinetics.

\section{The fast current is distinct from the A-current}

It has been previously reported that forskolin speeds the inactivation of the A-current in the bag cell neurons (Strong, 1984). This potassium current is activated and inactivated at more negative potentials than the two currents studied in this paper. One can investigate the A-current by removing resting inactivation with hyperpolarizing prepulse (to below $-75 \mathrm{mV}$ ). If the potential is then stepped to a value above $-50 \mathrm{mV}$, the A-cur- rent is observed as an outward current that activates and then inactivates. Since both the A-current and the fast current are inactivating potassium currents whose inactivation is more rapid after forskolin application, one might well ask if they are, in fact, the same current: At a holding potential of $-60 \mathrm{mV}$ (as used in the present experiments), could a small amount of A-current escape resting inactivation and give rise to the so-called fast current? Several observations argue against this hypothesis:

1. Steady-state inactivation of A-current is steeply voltagedependent and is $99.2 \%$ complete at a holding potential of -60 $\mathrm{mV}$ [when assayed with test depolarizations to $-25 \mathrm{mV}$ (Strong, 1984)]; the fast current is still present at holding potentials of $-40 \mathrm{mV}$.

2. During experiments in which forskolin was added to the bath or removed from the bath, we occasionally observed a (transient) situation in which the A-current inactivated rapidly but the fast current inactivated slowly, or vice versa.

3. In some cells $(<10 \%)$, the fast current was observed to be spontaneously fast-inactivating, before application of forskolin. Such cells still had slowly inactivating A-currents.

4. The relative amounts of A-current and fast current varied considerably from cell to cell.

5. If the fast current and the A-current were identical, one would expect that changing the holding potential to a value sufficiently negative to remove most A-current inactivation would simply enhance the inactivating portion of the outward current seen on depolarization (which we have so far attributed to the fast current). This is not the case, however. When the holding potential is changed to $-90 \mathrm{mV}$ (removing most of the resting inactivation of the A-current), a $200 \mathrm{msec}$ depolarization to $+30 \mathrm{mV}$ elicits outward currents with two distinct inactivating components (in contrast to the single component seen 


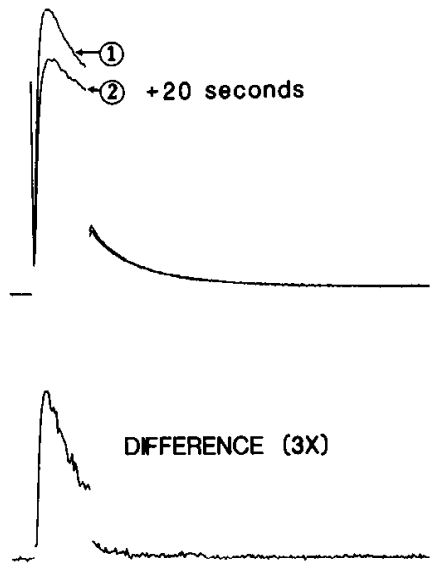

Figure 8. Inactivation of outward current with repetitive depolarizations. Current evoked by $30 \mathrm{msec}$ depolarization to $30 \mathrm{mV}(I)$ is superimposed on the current evoked by a second depolarization (2) given $20 \mathrm{sec}$ later. Both depolarizations were followed by repolarization to $-40 \mathrm{mV}$. The difference between the two records is shown below, at $3 \times$ larger current scale. The first depolarization was given at least 2 min after any previous depolarizations.

when the holding potential is $-60 \mathrm{mV}$; see Figs. 1 and 2). The faster of these two components (presumably the A-current) can be eliminated by returning the holding potential to $-60 \mathrm{mV}$ for $50-100 \mathrm{msec}$. The slower of these two components has an inactivation time course like that of the fast current. Its amplitude is enhanced when the holding potential is changed from -60 to $-90 \mathrm{mV}$, and in order to remove this enhancement, the holding potential must be returned to $-60 \mathrm{mV}$ for $10-30 \mathrm{sec}$. This indicates that the A-current and the fast current have very different kinetic properties.

\section{Inactivation with repetitive stimulation}

In many neurons (Thompson and Aldrich, 1980), including bag cell neurons (Kaczmarek and Strumwasser, 1981), one observes progressive broadening of spike width during a train of action potentials. Under voltage clamp, one can observe a progressive diminution of outward current with repetitive depolarizations. In Figure 8, the latter phenomenon is also seen in the dialyzed bag cell neurons. We observed that the complete recovery of outward current following a single depolarization is exceedingly slow: At a holding potential of $-60 \mathrm{mV}$, an interstimulus interval greater than $60 \mathrm{sec}$ is required to avoid inactivation with repetitive depolarizations (Fig. 8). In Dorid neurons, delayed outward current shows a similarly slow recovery from inactivation (Aldrich et al., 1979). In the bag cell ncurons, this slow recovery seems to be at least partly a property of the fast current. As in Figure 8, the difference current (i.e., the current during the initial depolarization minus the current during a subsequent depolarization), like the fast current, shows inactivation during a short depolarization. The phenomenon of slow recovery from inactivation was not seen in every cell studied with the protocol shown in Figure 8. Some cells with large amounts of the fast current showed little inactivation with repetitive depolarization at $20 \mathrm{sec}$ intervals, and, in other cells, the phenomenon tended to disappear during the course of the experiment. This may reflect cell-to-cell variability in the voltage dependence of recovery from inactivation, which is greatly speeded at more hyperpolarized holding potentials. Alternatively, the phenomenon may be particularly sensitive to intracellular dialysis.

Repetitive depolarizations with a much smaller interstimulus interval (e.g., 1-5 sec) caused a progressive reduction of the slow current (not shown). This phenomenon, unlike the slow recovery shown in Figure 8, was seen in all cells studied and showed no lability during the course of a single experiment.

\section{Discussion}

The main finding of this study is that delayed outward current in the bag cell neurons consists of two kinetically distinct components, both of which are functionally reduced by application of forskolin and theophylline. The slow current, $I_{\mathrm{k} 1}$, is reduced in amplitude by forskolin. The fast current, $I_{K 2}$, inactivates more rapidly after forskolin application. Taken together with the previously reported result that forskolin speeds the inactivation of the A-current (Strong, 1984), these results indicate that all three of the major voltage-dependent potassium currents in the bag cells are reduced by forskolin. In contrast, the calcium current in these cells is unaffected by elevations of cAMP, but is enhanced by activation of protein kinase- $C$ [the calcium/phosphatidylserine/diacylglycerol-dependent kinase (DeRiemer et al., 1985)]. We have not yet studied the effects of forskolin on the potassium current activated by calcium entry. Although the two currents described in this paper are clearly voltage-dependent, and are not activated by calcium entry under the conditions of our experiments, we cannot eliminate the possibility that one or the other of the currents may also have some form of calcium-dependent regulation.

Despite the fact that there are already three different currents known to be modified by cAMP in the bag cells, there remain several observations whose ionic bases have not yet been determined. One concerns the induction of a region of negative slope resistance by 8-benzylthio-cAMP (Kaczmarek and Strumwasser, 1984). To date, we have not been able to see this effect in dialyzed cells. The onset of membrane oscillations and the increase in membrane resistance (measured near the resting potential) seen during an afterdischarge, or during cAMP elevations in bag cells in primary culture, have also not yet been explained by voltage clamp data.

Delayed outward currents, carried by potassium, have been found in virtually every type of excitable cell (for reviews, see Hille, 1984; Thompson and Aldrich, 1980). Hille (1984) has pointed out that the term "delayed rectifier" has been used to describe an entire class of potassium channels that, though they are functionally similar, show considerable intra- and interspecies heterogeneity in their kinetic and pharmacological properties. Following Hille's usage, we would describe $I_{\mathrm{K} 1}$ and $I_{\mathrm{K} 2}$ as two kinetically distinct components of delayed rectification in the bag cell neurons. The existence of two separate components of delayed rectification in the same cell is somewhat unusual, but has bcen reported in cardiac Purkinje fibers (Noble and Tsien, 1969), skeletal muscle (Adrian et al., 1970), and frog node of Ranvier (Dubois, 1981). Examples of an inactivating component of delayed rectification (qualitatively similar to our $I_{\mathrm{K} 2}$ ) are discussed in the latter two papers; it is more common for delayed rectifier current to inactivate only with prolonged depolarization, as does $I_{\mathrm{K}}$ in the bag cell neurons. We refer to $I_{\mathrm{K} 2}$ as an inactivating component of delayed rectification, rather than as an A-current with an unusual voltage dependence, because, again following Hille's (1984) usage, we believe the term "A-current" to be more useful as a name for a functional class of potassium channels (namely, those that, by virtue of their voltage dependences, control interspike interval in repetitively firing cells) than as a name for all inactivating potassium channels. The literature does not yet reflect complete uniformity on these semantic issues, however. $I_{\mathrm{K} 2}$ is also distinct from the calcium-dependent inactivating outward currents that have been reported in a number of cells (i.e., MacDermott and Weight, 1982; Miledi, 1982; Mouneir and Vassort, 1975; Siegelbaum and Tsien, 1980), since it is present in cells dialyzed with 20 mM EGTA and remains when calcium current is blocked with $\mathrm{La}^{3+}$.

The two voltage-activated potassium currents discussed in this paper seem, by virtue of their time and voltage dependences, well situated for playing a role in the repolarization of the action 
potential. For comparison, the action potentials seen during the early phase of an electrically stimulated afterdischarge in situ have a width of $65 \mathrm{msec}$ and depolarize the cell to approximately +20 mV (Kaczmarek et al., 1982). In the present experiments, the net outward current (including both $I_{\mathrm{K} 1}$ and $I_{\mathrm{K} 2}$ ) during a depolarization to $+15 \mathrm{mV}$ was maximal at $70 \mathrm{msec}$; forskolin caused a marked reduction of the currents seen during this time period (see Fig. 4). Thus, the reduction of these two currents by cAMP could contribute to the enhancement of the width of action potentials seen during the natural bag cell afterdischarge. In this context, it is worth noting that $50 \mu \mathrm{M}$ forskolin (with 1 $\mathrm{mM}$ theophylline) elevates the cAMP concentration of intact bag cell clusters only 3-fold (Kauer and Kaczmarek, 1985). Since we saw partial reduction of both polassium currents with concentrations of forskolin as low as $5 \mu \mathrm{M}$, it seems not unreasonable to expect that some change in these currents will occur during a natural afterdischarge, when cAMP levels are also elevated approximately 3 -fold (Kaczmarek et al., 1978).

Several obser vations support the idea that forskolin was acting through the adenylate cyclase system. Few effects were seen without a phosphodiesterase inhibitor, and the same results were obtained using either of two such inhibitors with very different chemical structures. In one cell, no effect of forskolin and theophylline was seen when ATP and GTP were omitted from the intracellular solution. Moreover, similar reductions in net outward currents were seen by Kaczmarek and Strumwasser (1984) after extracellular application of membrane-permeant cAMP analogs.

There have been several reports of cAMP-mediated changes in the electrical properties of excitable tissues (for review, see Siegelbaum and Tsien, 1983). It is particularly interesting to compare the results reported here with those seen in other identified Aplysia neurons. In R15, a bursting cell, the anomalous rectificr is cnhanced by serotonin or by bath application of membrane-permeant cAMP analogs (Levitan and Adams, 1981). In sensory neurons, serotonin or intracellular injection of cAMP closes another type of potassium channel, the "S" channel (Siegelbaum et al., 1982). The A-current in these cells is unaffected by serotonin (Klein et al., 1982). Connor and Hockenberger (1984) report that intracellular injection of cAMP into R15 and certain other identified Aplysia neurons induces a novel inward sodium current. Although there are some methodological differences between the different experiments, this comparison of the effects of cAMP in different Aplysia neurons suggests that the cAMP-dependent regulatory enzymes are put to different and specific uses in different neurons of a given species. More direct evidence for this idea comes from Hockenberger and Connor (in press), who systematically compared various identified neurons of the mollusk, Limax, and found cell-specific differences in their responses to cAMP injection.

One major difference between the bag cell neurons and other Aplysia neurons studied is that, in the bag cells, cAMP effects are larger and involve multiple channels. This may be because the cAMP-initiated bag cell afterdischarge represents a complete change in the cells' properties, functioning as an $\mathrm{ON} / \mathrm{OFF}$ switch for egg-laying behavior, whereas in the other examples, cAMP controls a more subtle modification of the cell's ongoing activity.

The nature of cell specificity in the cAMP-dependent regulation of ion channels has not yet been elucidated. One possibility is that these differences result because some cells express channels that others lack altogether. A variant of this idea is that a particular electrophysiologically defined current may actually represent an entire family of related channel proteins. Thus, the A-current channel in the bag cells (which is regulated by cAMP) may be a slightly different protein than the A-current channel in sensory neurons (which is apparently not cAMPdependent). Genetic evidence for such families of proteins has been found in other systems. A second possibility is that reg- ulation of ion channels at cAMP utilizes intermediary (and channel-specific) enzymes, which differ from cell to cell. A third possible mechanism for two different cells with identical channels and identical cAMP-dependent regulatory enzymes is a differing subcellular localization of the cAMP-dependent enzymes, which could be near different substrates and/or channels. Molluscan nervous systems may provide interesting tests of these hypotheses.

\section{References}

Adams, D. J., S. J. Smith, and S. H. Thompson (1980) Ionic currents in molluscan soma. Annu. Rev. Neurosci. 3: 141-167.

Adrian, R. H., W. K. Chandler, and A. L. Hodgkin (1970) Slow changes in potassium permeability in skeletal muscle. J. Physiol. (Lond.) 208: 645-668.

Aldrich, R. W., P. A. Getting, and S. H. Thompson (1979) Inactivation of delayed outward current in molluscan neuronal somata. J. Physiol. (Lond.) 291: 507-530.

Connor, J. A., and P. Hockenberger (1984) A novel membrane sodium current induced by injection of cyclic nucleotides into gastropod neurones. J. Physiol. (Lond.) 354: 139-162.

DeRiemer, S. A., J. A. Strong, K. A. Albert, P. Greengard, and L. K. Kaczmarek (1985) Enhancement of calcium current in Aplysia neurones by phorbol ester and protein kinase C. Nature 313: 313-315.

Dubois, J. M. (1981) Evidence for the existence of three types of potassium channels in the frog ranvier node membrane. J. Physiol. (Lond.) 318: 297-316.

Hamill, O. P., A. Marty, E. Neher, B. Sakmann, and F. J. Sigworth (1981) Improved patch-clamp techniques for high-resolution current recording from cells and cell-free membrane patches. Pfluegers Arch. 391: 85-100.

Heller, E., L. K. Kaczmarek, M. W. Hunkapiller, L. E. Hood, and F. Strumwasser (1980) Purification and primary structure of two neuroactive peptides that cause bag cell afterdischarge and egg-laying in Aplysia. Proc. Natl. Acad. Sci. USA 77: 2328-2332.

Hille, B. (1984) Ionic Channels of Excitable Membranes, Sinauer, Sunderland, MA.

Hockenberger, P., and J. A. Connor (in press) Alteration of calcium conductances and outward current by cAMP in neurones of Limax maximus. J. Cell Mol. Neurobiol.

Hodgkin, A. L., and A. F. Huxley (1952) The components of membrane conductance in the giant axon of Loligo. J. Physiol. (Lond.) 116: 473-496.

Kaczmarek, L. K., and F. Strumwasser (1981) The expression of longlasting afterdischarge by isolated Aplysia bag cell neurons. J. Neurosci. 1: $626-634$

Kaczmarek, L. K., and F. Strumwasser (1984) A voltage clamp analysis of currents underlying cAMP-induced membrane modulation in isolated peptidergic neurons of Aplysia. J. Neurophysiol. 52: 340349.

Kaczmarek, L. K., K. Jennings, and F. Strumwasser (1978) Neurotransmitter modulation, phosphodiesterase inhibitor effects, and cAMP correlates of afterdischarge in peptidergic neurites. Proc. Natl. Acad. Sci. USA 75: 5200-5204.

Kaczmarek, L. K., K. R. Jennings, and F. Strumwasser (1982) An early sodium and a late calcium phase in the afterdischarge of peptidesecreting neurons of Aplysia. Brain Res. 238: 105-115.

Kauer, J., and L. K. Kaczmarek (1985) Peptidergic neurons of Aplysia lose their response to cyclic adenosine $3^{\prime}-5^{\prime}$-monophosphate during a prolonged refractory period. J. Neurosci. 5: 1339-1345.

Klein, M., J. Camardo, and E. R. Kandel (1982) Serotonin modulates a new potassium current in the sensory neurons that show presynaptic facilitation in Aplysia. Proc. Natl. Acad. Sci. USA 79: 5713-5717.

Kostyuk, P. G. (1984) Intracellular perfusion of nerve cells and its effects on membrane currents. Physiol. Rev. 64: 435-454.

Kupferman, I., and E. R. Kandel (1970) Electrophysiological properties and functional interconnections of two symmetrical neurosecretory clusters (bag cells) in abdominal ganglion of Aplysia. J. Neurophysiol. 33: 865-876.

Levitan, I. B., and W. B. Adams (1981) Cyclic AMP modulation of a specific ion channel in an identified nerve cell: Possible role for protein phosphorylation. Adv. Cyclic Nucleotide Res. 14: 647-653.

Levitan, I. B., and J. Norman (1980) Different effects of cAMP and 
cGMP derivatives on the activity of an identified neuron: Biochemical and electrophysiological analysis. Brain Res. 187: 415-429.

MacDermott, A., and F. Weight (1982) Action potential repolarization may involve a transient, $\mathrm{Ca}^{++}$-sensitive outward current in a vertebrate neuron. Nature 300: 185-188.

Miledi, R. (1982) A calcium-dependent transient outward current in Xenopus laeis oocytes. Proc. R. Soc. Lond. [Biol.] 215: 491-497.

Mournier, Y., and G. Vassort (1975) Evidence for a transient potassium current dependent on $\mathrm{Ca}^{++}$influx in crab muscle fiber. J. Physiol. (Lond.) 251: 609-625.

Noble, D., and R. W. Tsien (1969) Outward membrane current in the plateau range of potential in cardiac Purkinje fibres. J. Physiol. (Lond.) 200: $205-231$.

Seamon, K. B., W. Padgett, and J. W. Daly (1981) Forskolin: Unique diterpene activator of adenylate cyclase in membranes and in intact cells. Proc. Natl. Acad. Sci. USA 78: 3363-3367.

Siegelbaum, S. A., and R. W. Tsien (1980) Calcium-activated transient outward current in cardiac Purkinje fibres. J. Physiol. (Lond.) 299: 485-506.
Siegelbaum, S. A., and R. W. Tsien (1983) Modulation of gated ion channels as a mode of transmitter action. Trends Neurosci. 6: 307312.

Siegelbaum, S. A., J. S. Camardo, and E. R. Kandel (1982) Serotonin and cAMP close single $\mathrm{K}^{+}$channels in sensory neurones. Nature 299: 413-417.

Strong, J. A. (1984) Modulation of potassium current kinetics in bag cell neurons of Aplysia by an activator of adenylate cyclase. J. Neurosci. 4: 2722-2783.

Strumwasser, F. L., L. K. Kaczmarak, A. Y. Chiu, E. Heller, K. R. Jennings, and D. P. Viele (1980) Peptides controlling behavior in Aplysia. In Peptides: Integrators of Cell and Tissue Functions, F. E. Bloom, ed., pp. 197-218, Raven, New York.

Thompson, S. H., and R. A. Aldrich (1980) Membrane potassium channels. In The Cell Surface and Neuronal Function, C. W. Cotman, G. Poste, and G. L. Nicolson, eds., pp. 50-78, Elsevier/North-Holland, Amsterdam. 